the meantime, genetic testing proved homozygosity for the pathogenic variant of the SPAG1 gene.

The treatment of PCD is challenging because of low evidence-based recommendations. However, this case report shows that with regular monitoring and therapy adjustments depending on the circumstances, it is possible to maintain the airways state and pulmonary function at a satisfactory level even when the profound chronic lung changes exist.

\section{ABNORMAL LOCALISATION OF THE RADIAL SPOKE HEAD PROTEIN 4 AS A CAUSE OF PRIMARY CILIARY DYSKINESIA- CASE REPORT}

Marijana Odobašić*, Marija Pečnjak, Blaženka Kljaić Bukvić, Mario Blekić, Ivan Pavić. Opća bolnica 'Dr.Josip Benčević', Slavonski Brod

\subsection{6/archdischild-2021-europaediatrics.416}

Primary ciliary dyskinesia (PCD) is a rare genetic heterogeneous disease with autosomal recessive inheritance. The estimated prevalence is 1:10 000 to 1:40 000 live-born. To date, more than 40 different genes where identified to be involved. The most common form of PCD is defects in the outer dynein arms and radical spokes. PCD is characterized by chronic upper and lower respiratory tract disease, infertility, neurological manifestations and abnormality in left-right asymmetry. The severity of disease varies between patients. When growing up, symptoms become more pronounced with the presence of destructive changes in the lungs, bronchiectasis, and the development of respiratory failure.

In this report we present a brother and sister, from young, healthy parents, who have recurrent respiratory symptoms and respiratory infections from infancy: chronic cough, recurrent bronchopneumonia and bronchitis. The first medical examinations, hospitalisations and laboratory testing for cause of recurrent infections were done in infancy. The laboratory findings were inconclusive. In May 2018., the boy, aged 11, is underfed (BMI $14.39 \mathrm{~kg} / \mathrm{m} 2,5$. ct) with clinical problems of chronic cough, and auscultator findings of crackles. We done the laboratory examination and tried to found out the underlining cause. The laboratory findings reveal no increase in inflammatory tests, negative microbiological samples, with the exclusion of humoral and cellular immunodeficiency's, sarcoidosis, aspergillosis, tuberculosis, heart disease and gastroesophageal reflux. We observed allergic sensitization, obstructive changes in pulmonary function test and bronchial hyperreactivity. High resolution computed tomography (HRTC) of the chest showed bronchiectasis in the lower lobe of the left and middle lobe of the right lung. Pulmonary function tests demonstrated obstructive ventilation functional impairment. Diagnostic bronchoscopy with mucosal sampling was performed. The immunofluorescence test showed RSPH9 abnormal localization in the ciliary axonemes. This finding confirms the diagnosis of PCD. At the same time, we follow up the boy's younger sister, at the age of 8 , with the similar clinical symptoms. Pulmonary function tests showed obstructive-restriction functional impairment, while the HRCT scan of the chest revealed atelectasis and bronchiectasis in the segments of the lower pulmonary lobe to the right and $\mathrm{V}$ segments to the left. According to the PID diagnosis in older brother, we presumed that girl has the same disease, respectively.

Primary ciliary dyskinesia is a rare disorder with no etiologic treatment.
Early diagnosis is important for introduction of early pulmonary rehabilitation and management of exacerbations in order to preserve the pulmonary parenchyma for as long as possible

\section{THE EFFECT OF NIV ON THE CLINICAL OUTCOME IN SEVERAL DIFFERENT PROFILES OF PEDIATRIC PATIENTS WITH CHRONIC RESPIRATORY FAILURE}

A Miculinic*, T Savic-Jovanovic, A Zupan, D Heli Litvic, SA Anzic, Z Vlasic Loncaric, M Turkalj. Srebrnjak Children's Hospital

10.1136/archdischild-2021-europaediatrics.417

Noninvasive ventilation (NIV), refers to the administration of ventilatory support without the use of intubation andis a widely used and efficient method for treating patients with chronic respiratory insufficiency. Recent studies show that the spectrum of patients who could be candidates for NIV may require further evaluation, because, if started as early as possible, patients could benefit from less complications and a better quality of life.

This retrospective study included 7 patients with chronic respiratory insufficiency due to neurological and respiratory disorders as part of a complex genetic disorder which were followed and treated at Srebrnjak Children's Hospital and ultimately received NIV as the main therapy of choice for respiratry failure. Laboratory findings and clinical status,as well as incidence of respiratory symptoms and freaquency of hospital admissions were analized.

All of our patients fulfilled diagnostic criteria for chronic respiratory failure (requiring dayly oxygen support, having baseline $\mathrm{SaO} 2<88 \%$ on room air or pCO2 $>50$ with a normal $\mathrm{pH}$ value).

Of our 7 patients $2(28,6 \%)$ were female and $5(71,4 \%)$ were male. Only one patient had no confirmed genetic diagnosis while $6(85,7 \%)$ had proven chromosomal aberations or genetic mutations. The most frequently diagnosed respiratory difficulties were recurrent respiratory infections of the upper airways $(\mathrm{N}=7,100 \%)$, lower airways with bronchial obstruction $(\mathrm{N}=5,71,4 \%)$ and recurrent pneumonias $(\mathrm{N}=2,73,4 \%)$.

Results show that all of the included patients had a better respiratory status (measured by pulse oxymetry and acid-base status) and a signifficant decrease in the frequency of respiratory infections was also observed.

We conclude that noninvasive ventilation is the therapy of choice for children with chronic respiratory insufficiency. We suggest that NIV should be started as early as possible after the initial diagnostic procedures were performed and the clinical and diagnostic criteria have been met. The indications for NIV should be further extended as to prevent severe complications in patients with chronic diseases that affect respiratory muscle strength and would also be beneficial for their quality of life.

\section{CYSTIC ADENOMATOID LUNG MALFORMATION - CAUSE OF RECURRENT PNEUMONIA}

Marko Bašković*, Mirko Žganjer, Davor Ježek, Ante Čizmić. Children's Hospital Zagreb

10.1136/archdischild-2021-europaediatrics.418 